\title{
Ruta biocultural de conservación de las semillas nativas y criollas en el territorio indígena de Puracé, Cauca
}

\section{Biocultural route for the conservation of native and creole seeds in the indigenous territory of Puracé, Cauca}

\author{
Marta Elena Montaño ${ }^{1}$; Olga Lucía Sanabria-Diago²; Ricardo Manzano ${ }^{3}$; Oswaldo Quilindo ${ }^{4}$ \\ 1'Ing. Civil, M.Sc., Ph.D.(c). Fundación Universitaria de Popayán, Departamento de Ecología. Popayán - Cauca, Colombia; e-mail: mmontano@unicauca.edu.co; \\ (D) https://orcid.org/0000-0002-4940-7624 \\ ²Licenciada en Biología y Química, M.Sc., Ph:D., Pos. doc. Universidad del Cauca. Popayán - Cauca, Colombia; e-mail: oldiago@unicauca.edu.co; D https://orcid. \\ org/0000-0001-6905-1808 \\ ${ }^{3}$ Bachiller, Comunero del Resguardo de Puracé. Puracé - Cauca, Colombia; e-mail: rialma3@hotmail.com; (D) https://orcid.org/0000-0003-2600-4715 \\ ${ }^{4}$ Tecnólogo Ambiental, Comunero del Resguardo de Puracé. Puracé - Cauca, Colombia; e-mail: oswaldo7728@hotmail.com; (D) https://orcid.org/0000-0003-2675- \\ 3898
}

Cómo citar: Montaño, M.E.; Sanabria-Diago, O.L.; Manzano, R.; Quilindo, O. 2021. Ruta biocultural de conservación de las semillas nativas y criollas en el territorio indígena de Puracé, Cauca. Rev. U.D.C.A Act. \& Div. Cient. 24(1):e1771. http://doi.org/10.31910/rudca. v24.n1.2021.1771

Artículo de acceso abierto publicado por Revista U.D.C.A Actualidad \& Divulgación Científica, bajo una licencia Creative Commons CC BY-NC 4.0

Publicación oficial de la Universidad de Ciencias Aplicadas y Ambientales U.D.C.A, Institución de Educación Superior Acreditada de Alta Calidad por el Ministerio de Educación Nacional.

Recibido: noviembre 23 de $2020 \quad$ Aceptado: mayo 5 de $2021 \quad$ Editado por: Ingeborg Zenner de Polanía

\section{RESUMEN}

Buscar la conservación de los sistemas productivos tradicionales en el territorio de Puracé implicó identificar los principales factores que los han afectado y definir estrategias, que posibiliten su conservación. En el resguardo, el esfuerzo de conservación, se realiza a través de una organización, de hecho, que pertenece al cabildo indígena, denominada custodios de semillas. Por esta razón, el trabajo se focalizó con ellos, con quienes se realizaron talleres, entrevistas y trabajo de campo, que involucraron observación participante, etnografías, caracterización etnobotánica y análisis cartográficos, lo que llevó a generar una Ruta biocultural de conservación de las semillas nativas y criollas. Se identificaron 156 semillas, que fueron etnoclasificadas, 90 de uso culinario, 37 medicinales, 16 usadas para alimento y medicina y 13 con otros usos; los factores bioculturales que afectan la conservación, se agruparon en 5 categorías; el relevo generacional es el principal factor cultural, sobre el cual, es necesario trabajar para la conservación de las semillas nativas y criolla, por lo que constituye el eje estructurante de la Ruta que se propuso.

Palabras clave: Revitalización; Conocimiento Tradicional; Custodios de semillas.

\section{ABSTRACT}

Seeking the conservation of traditional production systems in the Puracé reservation, implied identifying the main factors that have affected them and defining strategies that enable their conservation. In the reservation, the conservation effort is carried out through an organization in fact that belongs to the indigenous council, called 
custodians of seeds. For this reason, the work was focused on them, with whom workshops, interviews and field work were carried out that involved participant observation, ethnographies, ethnobotanical characterization and cartographic analysis, leading to establish the need to generate a biocultural route for the conservation of native seeds. and Creoles. 156 seeds were identified that were ethnoclassified, 90 for culinary use, 37 medicinal, 16 used for food and medicine, and 13 for other uses; The factors that affect conservation were grouped into 5 categories, the generational change is the main factor on which it is necessary to work is conservation, constituting the structuring axis of the Route that was proposed.

Keywords: Revitalization; Traditional Knowledge; Custodians of seeds.

\section{INTRODUCCIÓN}

Las semillas nativas son aquellas que su centro de origen se encuentra en la misma región donde se cultivan (Harlan, 1971) y las criollas, aunque no son originarias del territorio, los agricultores las adaptan en sus huertas, a las condiciones ambientales (Gutiérrez \& Fitting, 2016) e intereses antropocéntricos locales. Su pérdida causa preocupación mundial, porque además de la reducción de especies, variedades o variantes cultivadas (Casas \& Parra, 2007), a tasas hasta 10.000 veces mayor que la extinción natural (Casas et al. 2016), también reduce la diversidad genética.

En Colombia, a partir de 1960, se impulsó la revolución verde, por medio de los programas de asistencia técnica, que llevaron a la sustitución de variedades locales por otras de mayor rendimiento productivo. Este hecho desvalorizó el conocimiento tradicional, promoviendo la disminución de la agrobiodiversidad en los territorios, al desestimar el valor científico de la domesticación y del manejo de las semillas nativas y criollas (Forero, 2010).

En el resguardo de Puracé, que corresponde a un territorio colectivo de 21.870ha (ANT, 2018; IGAC, 2016), localizado en la zona altoandina, al sur occidente de Colombia, habitado por 5.173 indígenas del pueblo Kokonuko (CIP, 2020), se ha reportado la transformación de sistemas diversificados a homogéneos (Valencia et al. 2017), que llevó a la pérdida de semillas de variedades de ulluco (Ullucus tuberosus Caldas), papa (Solanum tuberosum L.) y maíz (Zea mays L.) (Piso et al. 2019).

La siembra intensiva de cultivos comerciales, como papa y la producción de leche, favorecieron la incursión de variedades de alto rendimiento, que redujeron los sistemas de producción tradicionales (Jimeno \& Triana, 1985). Esto provocó, que el ulluco, localmente conocido como solferino y el maíz matambre, dejaran de producirse en el territorio; se pasó de 70 variedades de papa que se sembraban a mitad del siglo XX, a 4 variedades, que componen los sistemas comerciales de la actualidad (Caldon et al. 2019).

En la medida que se presentó el fortalecimiento de los sistemas comerciales, apareció la preocupación de la organización indígena por la pérdida de variedades nativas y criollas. Es así, que en la década de los 90 del siglo pasado, se inicia un proceso de recuperación de semillas, con el objetivo de conservar los sistemas tradicionales, semillas nativas y criollas, a través del fortalecimiento de huertas tradicionales (Mazabuel, 1999).

Desde el 2006, se da peso político a la conservación y la recuperación de semillas nativa y criollas, al incorporar este tema en los congresos internos, en los cuales, la comunidad en pleno, se reúne para tomar decisiones, que den orientación política organizativa y establezcan la ruta de trabajo y fortalecimiento de los procesos sociales, culturales, económicos y ambientales, para la pervivencia como pueblo Kokonuko.

Los cinco congresos internos realizados en el territorio, determinaron acciones de recuperación y de valoración de los conocimientos tradicionales, el incremento de las áreas con cultivos tradicionales, la recuperación de semillas nativas y criollas y no permitir el ingreso de Organismos Genéticamente Modificados -OGM- al territorio.

En el 2010, con el objetivo de conservar los sistemas tradicionales, se establece una organización al interior del cabildo, denominada custodios de semillas, que son personas con conocimientos tradicionales, que se dedican de tiempo parcial o completo, a conservar, cuidar, cosechar y compartir las semillas nativas y criollas (Chacón \& García, 2016).

La red cuenta, actualmente, con 14 experiencias de producción tradicional, manejadas por la misma cantidad de custodios de semillas, con edades que oscila entre los 50-90 años. A pesar del gran conocimiento y la riqueza de agrodiversidad existente en las parcelas de los custodios, el potencial de relevo generacional no se ha logrado, poniendo en riesgo los esfuerzos realizados para su conservación y recuperación.

El custodio tiene en su parcela un laboratorio de experiencias, donde cuida, produce, maneja y adapta estas semillas, crea relaciones bioculturales, a través de las prácticas agrícolas de fitomejoramiento, que generan y fortalecen el conocimiento tradicional, además de mantener viva la cultura local, la conservación e intercambio de variedades nativas y criollas (Thiele, 1999; Badstue et al. 2006; Hermann et al. 2009).

Al no producir e intercambiar estas semillas, se pierden también las redes y los procesos asociados (Zent \& Maffi, 2009), además de reducir los sistemas de conservación in situ (Dzib-Aguilar et al. 2016), de alto valor cultural (Sanabria, 2001). Las redes y los procesos de conservación de semilla tradicionales y criollas enfrentan problemas asociados a las condiciones climáticas, políticas, económicas y sociales (Shiva, 2020) que, en oportunidades, rompen la transmisión de conocimiento, propiciando dificultades de relevo generacional en la conservación.

Con la problemática de la no trasmisión del conocimiento tradicional, en el proceso de conservación de semillas, en el presente documento, se recogen las causas que han afectado la conservación biocultural y las estrategias de conservación, en una Ruta biocultural de conservación de las semillas nativas y criollas. 
La ruta acordada involucra la conservación con perspectiva biocultural, a través de la valoración de los sistemas tradicionales, su importancia en la conservación de los conocimientos asociados a estos sistemas, además de la reflexión y el análisis de acciones requeridas, para garantizar el trabajo intergeneracional.

\section{MATERIALES Y MÉTODOS}

La metodología de la ruta incorpora el análisis de la biodiversidad, a través de métodos etnobotánicos cuantitativos y cualitativos, con participación permanente de los custodios de semillas. El análisis en el resguardo de Puracé, sobre la necesidad de conservación biocultural de las semillas nativas y criollas, así como de los conocimientos asociados a estas, que constituyeron la primera experiencia que vincula, de forma integral, la conservación de la agrobiodiversidad y del conocimiento tradicional, en este territorio indígena.

Este proceso, se dio con reuniones de trabajo de campo y mapeo comunitario, con un equipo de diálogo intercientífico (Sletto et al. 2013), que permitió identificar el número de especies y las variedades por parcela, con el fin de georreferenciar la biodiversidad de cada parcela.

Con el fin de establecer el vínculo entre la agrobiodiversidad y los conocimientos asociados, que son objeto de conservación, se realizó la caracterización etnobotánica de las semillas conservadas y de los conocimientos usados para la producción, en las parcelas de los custodios de semillas, para lo cual, se empleó el manual de etnobotánica para trabajos de campo (Sanabria, 2001).

A partir de entrevistas con los custodios participantes (Malinowski, 1986) y talleres de trabajo con mayores y niños, se realizó el análisis de los factores que han afectado el proceso de transmisión de conocimiento y su impacto sobre la conservación de las semillas.

Los resultados, se complementaron con las experiencias de conservación, entre el grupo de Etnobotánicos Latinoamericanos (GELA), de la Universidad del Cauca y los custodios de semilla del resguardo de Puracé, que generó las estrategias de la ruta biocultural de conservación, que contó, además, con la participación de los custodios de semilla del resguardo de Puracé. Todas las acciones realizadas tuvieron lugar entre mayo del 2019 y junio del 2020.
Es importante resaltar que los datos de este trabajo contaron con el consentimiento previo, libre e informado de la comunidad, que se materializó, a través de un permiso otorgado por el cabildo y un convenio firmado entre la Universidad del Cauca y el Cabildo Indígena de Puracé, por medio de su gobernador principal. De igual manera, todo el proceso de caracterización etnobotánica y de identificación de conocimientos tradicionales, se suscribió al código de ética de la Sociedad Latinoamericana de Etnobiología (Cano-Contreras et al. 2016).

\section{RESULTADOS Y DISCUSIÓN}

Se georreferenciaron y mapearon comunitariamente 14 parcelas de custodios de semillas (Figura 1), con áreas que oscilan entre los 283,7 y los 16,134,1 $\mathrm{m}^{2}$. El 78\% de las parcelas, se encuentran ubicadas en la formación vegetal de bosque andino (Tabla 1).

En estas parcelas, se caracterizaron 155 variedades clasificadas por nombres comunes (Anexo), de las cuales, 54 son hortalizas, 40 tubérculos, 20 frutales, 9 cereales, 17 herbáceas y 15 son ornamentales. Si bien se encuentran 54 semillas que pertenecen a las hortalizas, la importancia de uso mostró que para la comunidad tiene mayor relevancia los tubérculos y los cereales, debido a que a estas categorías pertenecen las semillas nativas y aquellas que tienen mayor vínculo con los sistemas de alimentación y medicina propios.

De las 40 variedades de tubérculos, 20 son de papa; de los cereales, todos corresponde a variedades de maíz (9), lo que evidencia la importancia de estas dos especies.

Del total de variedades, 90 son de uso culinario, 37 medicinales, 16 usadas para alimento y medicina y 13 con otros usos (ornamental y artesanal), es decir, que los principales intereses del pueblo Kokonuko son el alimento y la salud.

$\mathrm{Al}$ analizar la distribución de plantas, se observa que las parcelas cuentan con una diversidad de especies, que va desde 14 hasta 55 variedades por parcela, teniendo en cuenta que las áreas son pequeñas; esto da cuenta de sistemas productivos de policultivos altamente diversos, en los que las semillas nativas no tienen arreglos productivos definidos, en tanto que las criollas sí se organizan en eras y terrazas.

Tabla 1. Distribución de parcelas por formación vegetal.

\begin{tabular}{|c|l|c|c|}
\hline \multirow{2}{*}{ No. parcelas } & \multicolumn{1}{|c|}{ Formación Vegetal } & \% Parcelas & Área $\left(\mathbf{m}^{2}\right)$ \\
\hline 2 & bosque subandino & 14,29 & 8620,61 \\
\hline 11 & bosque andino & 78,57 & 52388,03 \\
\hline 1 & bosque alto andino & 7,14 & 2060,62 \\
\hline
\end{tabular}




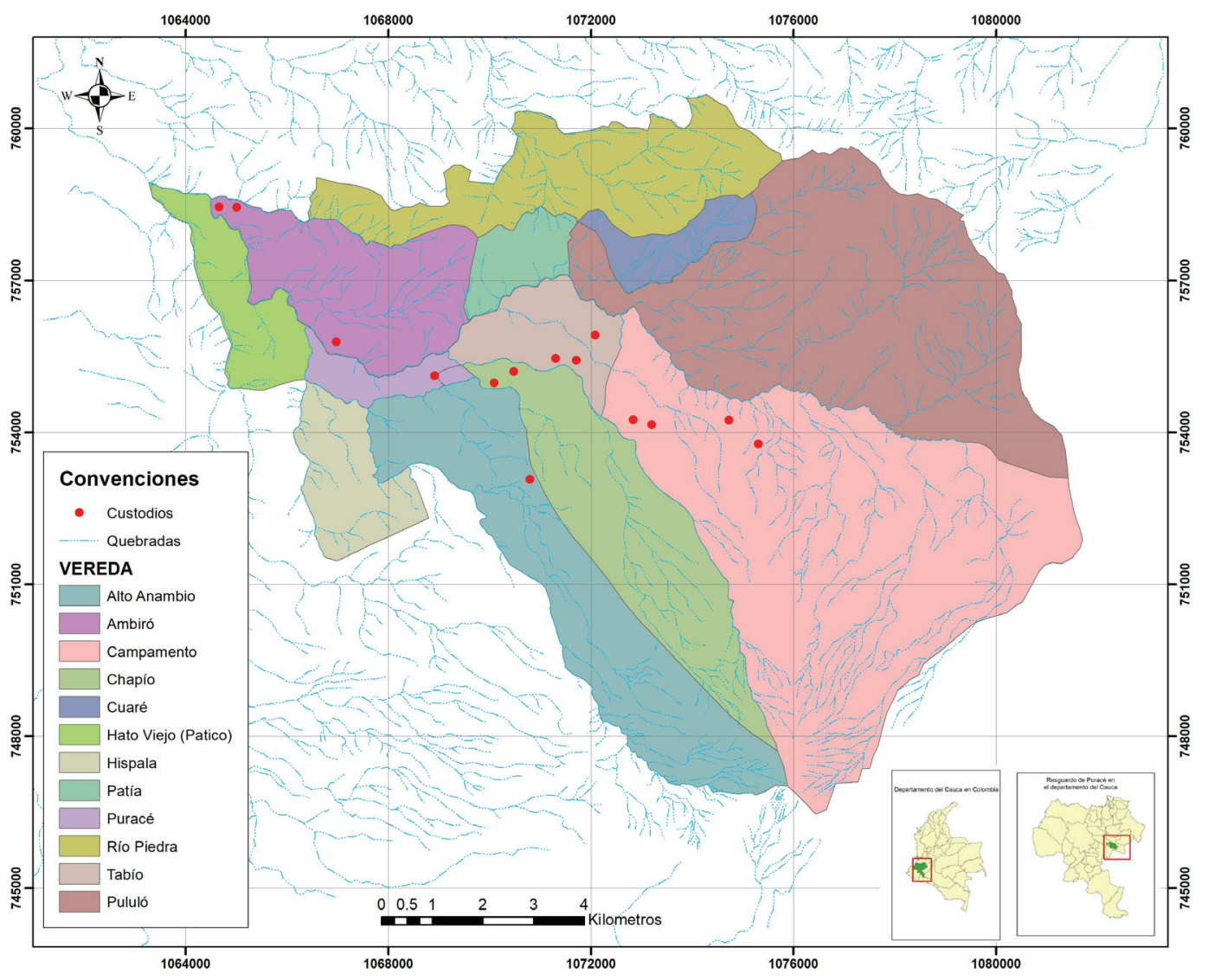

Figura 1. Mapa de ubicación de parcelas de custodios de semillas.

Tal como se observan en las figuras 2 y 3 , no existe una correlación entre la altitud o el tamaño de la parcela con el número de variedades cultivadas en estas. Por consiguiente, la diversidad está principalmente asociada a decisiones tomadas por los custodios de semilla, más que por las condiciones ambientales, relacionadas con la altitud y la superficie de la parcela custodio.

Para las decisiones del momento de siembra y los arreglos productivos, los custodios se basan en el uso de bioindicadores. Se identificaron 13 relacionados con el clima (Tabla 2), de los cuales, tres se encuentran asociados al uso de la luna y el sol, para la toma de decisiones sobre la siembra; cinco, se refieren a usos de bioindicadores, para el manejo del tiempo atmosférico y cinco, al conocimiento de manejo de plagas y de enfermedades. Todos los custodios, cumplido el ciclo productivo, rotan las variedades dentro de la parcela, reemplazando una semilla por otra o, incluso, por otro cultivo, el cual, se establece de acuerdo con las condiciones climáticas y las necesidades que tiene el custodio en el momento de la nueva siembra; tomado de las entrevistas a custodios en el 2019.

El conocimiento de amplia diversidad de variedades de semillas usadas, sus usos y formas de siembra, nos muestran que las parcelas reúnen complejas e integradas cosmovisiones (cosmos), alrededor de las cuales, se han generado conocimientos (corpus), que han permitido la generación de prácticas productivas (praxis) (Toledo, 2005), donde los custodios de semillas establecen las relaciones para el uso o manejo de la naturaleza y sus procesos.

Para establecer la razón por la que el relevo generacional de los custodios no se ha logrado, es importante partir, tanto de la comprensión de la reflexión propia de los mismos custodios como del análisis de lecciones aprendidas de los esfuerzos que el cabildo ha realizado, en este sentido.

Por tanto, una vez caracterizadas las semillas conservadas en las parcelas, se estableció la percepción de los custodios sobre las causas por las que no se ha generado la posibilidad de contar con el relevo generacional. El resultado mostró que los principales factores que han llevado a desestimular la siembra de semillas nativas y criollas, en orden de importancia, son: la pérdida de importancia de uso de las semillas, la homogenización de la comida asociada a la pérdida de platos tradicionales, la disminución del uso de plantas medicinales, el desestimulo que tuvo la agricultura en el territorio frente a la minería y ganadería, la falta de canales de comercialización y la falta de conocimiento de los jóvenes que estas semillas están presentes en el territorio. 


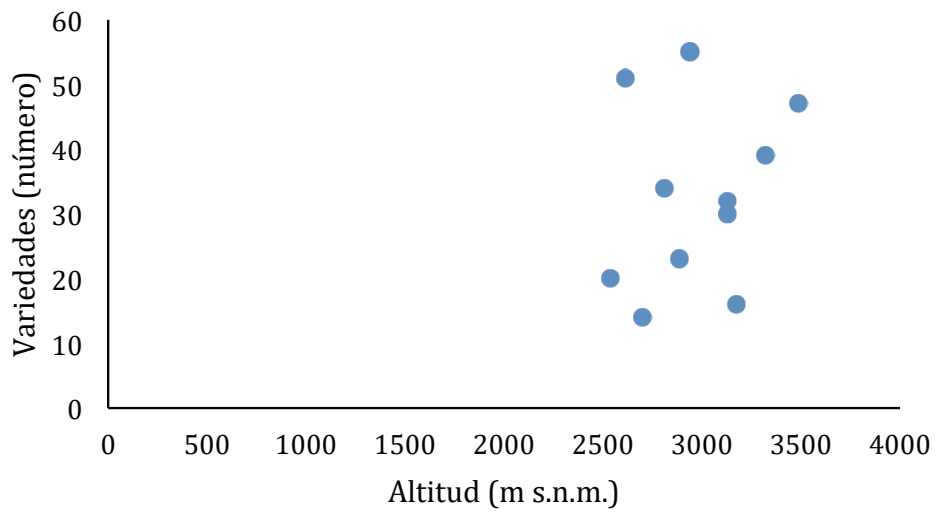

Figura 2. Distribución de número de variedades (especies - variedades), por superficie de las parcelas custodio.

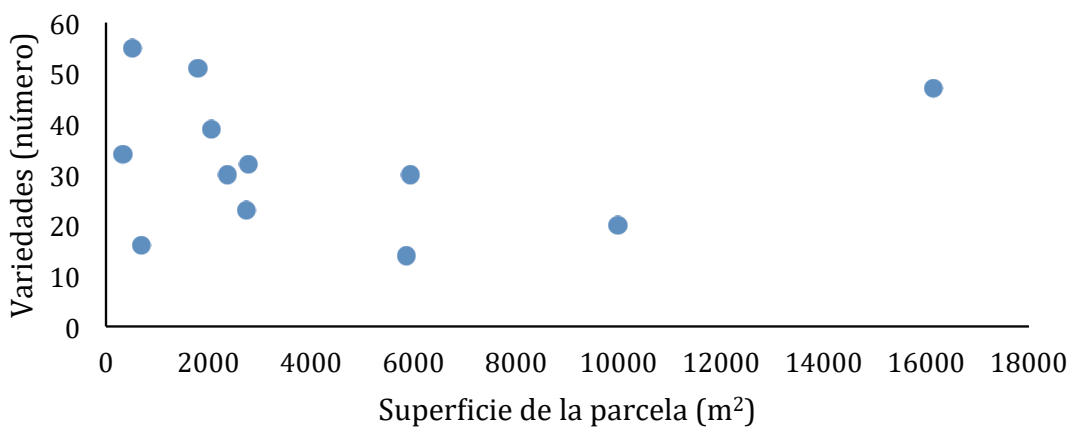

Figura 3. Número de variedades (especies - variedades) por parcela, de acuerdo con la altitud.

Tabla 2. Prácticas culturales relacionadas con las condiciones de clima.

\begin{tabular}{|c|c|}
\hline Proceso cultural & Tipo de relación \\
\hline Relación de la luna con las actividades agrícolas & Las labores se realizan si la luna acompaña o no el territorio \\
\hline Momento de la luna para la castración de animales & Los animales no se castran en luna nueva porque el animal se hincha \\
\hline Relación de la luna con el momento de preparación de terreno & $\begin{array}{l}\text { En luna nueva no se puede preparar el terreno, la luna apta para esto } \\
\text { es luna llena }\end{array}$ \\
\hline Golondrinas & De acuerdo con el tamaño indican llegada de invierno o verano \\
\hline Hormigas voladoras & $\begin{array}{l}\text { Cuando aparecen hormigas voladoras cafés, se puede sembrar. Indi- } \\
\text { can que va a llegar tiempo de lluvias }\end{array}$ \\
\hline La lombriz loca & $\begin{array}{l}\text { Las lombrices en la casa brincando y mueren, va a llegar verano, por } \\
\text { consiguiente, no se puede sembrar }\end{array}$ \\
\hline $\begin{array}{l}\text { El paletón, pájaro alargado, de plumaje amarillo, verde, rojo y negro. } \\
\text { Pico largo y curvo de colores verde, rojo y negro }\end{array}$ & $\begin{array}{l}\text { Cuando cantan pronostican que va a venir sequía interestival en la } \\
\text { zona alta }\end{array}$ \\
\hline $\begin{array}{l}\text { Las tijeretas, aves negras de collar blanco y cola alargada en forma de } \\
\text { tijera }\end{array}$ & Pronostican cambio de estado de tiempo \\
\hline Uso de trampas para plagas & Para el control de plagas \\
\hline Uso de orines de curíes (Cavia porcellus) u otras especies & Ahuyenta las ardillas \\
\hline Fumigar con leche, suero y miel de purga (melaza) & Para el control de la Gota (Phytophthora infestans) \\
\hline Colocar ollas con agua dentro del cultivo & Para la chamusquina (chinche monalonion) \\
\hline Humear la parcela & Para la helada \\
\hline
\end{tabular}


Esta información, junto a la obtenida en los talleres comunitarios con los mayores, realizados en el 2018 y al análisis de las lecciones aprendidas de los proyectos desarrollados entre el 2010 y la actualidad, evidencian que los jóvenes no producen en sistemas tradicionales, porque los perciben como insostenibles económicamente y por la falta de estrategias, que los vinculen a este proceso.

En diálogo con los mayores, en los talleres comunitarios, se plantea que muchas de las semillas que se conservan en los sistemas tradicionales no se reconocen por parte de los jóvenes, ni se tiene experiencia de cómo manejarlas. En las entrevistas, los mayores relatan que los jóvenes poco usan majua (Vallea stipularis) y oca (Oxalis tuberosa) en la alimentación, tampoco se siembra ulluco solferino, que es el que se considera nativo; las variedades de papa tradicionales que se conservan en las parcelas de los custodios han sido reemplazadas por las variedades mejoradas de papa suprema y única; no se siembra trigo, no se produce planta silvestre denominada "matambre" (Kohleria sp.), papa Rusia, papa San Jorge, que se cultivaban entre 1950-1980 (Valencia et al. 2017); la papa guata manzana, la papa blanca y la papa rosada, se han perdido, también el maíz Yucatán y la cebada (Proclama del Cauca, 2016).

Para dar respuesta a estas dificultades, la estrategia acordada, plantea la necesidad de garantizar la conservación de los conocimientos y su transmisión, a través de cinco parcelas piloto, que serán ubicadas en el territorio, a través de las cuales, se impulsará las innovaciones orientadas a fortalecer los sistemas de medicina tradicional y de alimentación propia.

Para garantizar el reconocimiento del saber tradicional de los mayores, que por cuestiones de salud deben dejar de cultivar la parcela y basados en la efectividad que ha demostrado la metodología de campesino a campesino en diferentes experiencias (Holt-Giménez, 2006), se propone crear una nueva categoría, denominada custodios de conocimientos, cuya función será el dar acompañamiento y promotoría en las cinco nuevas experiencias. Otros lineamientos de importancia, para continuar con el proceso de conservación biocultural, son:

- Elaborar una cartilla, que recoja las experiencias de los custodios de semillas.

- Generar diálogo intercientífico sobre las plantas medicinales, para identificar otros usos.

- Realizar estudios de control biológico, para generar estrategias propuestas de manejo, para las principales plagas presentadas.

- Generar bancos de semillas bajo condiciones de almacenamiento ambiental y culturalmente adecuadas, para cada especie vegetal.

- Establecer reglas para el control de las semillas durante el proceso de intercambio, almacenamiento y manejo, previo a la siembra, para evitar la propagación de plagas y de enfermedades.

- Recoger y sistematizar acciones culturales, para la adaptación al cambio climático.

- Capacitaciones en procesos de innovación en gastronomía con productos propios y en medidas, para generar nuevos platos con las especies nativas y criolla.

- Realizar estudios de caracterización e identificación de presencia de transgénicos.
- Buscar mercados solidarios, en los cuales, se pueda comercializar la producción, a precios justos.

- Fortalecer el sistema de Participación en Garantías.

- Vincular el trabajo social de las sedes e instituciones educativas con el cuidado de las parcelas tradicionales

Estas acciones, se trabajarán a partir de procesos de capacitación y de espacios de intercambio intergeneracional de semillas y de conocimientos, orientadas al trabajo en conjunto en parcelas, al impulso de capacitación con la metodología de campesino a campesino, que permita vincular a los mayores y jóvenes, a través de nuevas parcelas, para custodiar semillas.

Es fundamental, que el cabildo y sus cuadros de apoyo avancen en: a) La recuperación de comida tradicional, a través de vincular a las instituciones educativas, con el fin de dirigir un proceso de concientización y de revitalización de la importancia de las semillas, para usos alimentarios y la orientación de la participación de los jóvenes a las parcelas de los custodios, por medio del trabajo social, que se debe cumplir en los últimos años de escolaridad y, b) La priorización de la investigación sobre innovaciones gastronómicas, que incluyan productos de semillas nativas y criollas, objeto de conservación.

Es importante fortalecer el Sistema de Salud Indígena Propio, SISPI, al promover la investigación de innovación en el uso de las plantas medicinales, en la transformación de éstas y en fortalecer rituales asociados a la conservación de las semillas, con el fin de recuperar el uso de plantas de importancia, por su uso medicinal.

Se propone proyectar el trabajo permanente por medio de mingas comunitarias entre jóvenes y mayores, con el fin de recuperar el vínculo de las nuevas generaciones con el proceso de conservación de las semillas, propiciando espacios de intercambio de saberes, que estrechen los vínculos intergeneracionales. Priorizar la investigación en nuevos usos en el territorio de las semillas tradicionales y criolla, procesos de transformación para generar pequeñas microempresas y nuevos mercados agroecológicos, con el fin de estimular la producción.

La agricultura convencional invisibilizó y marginó los procesos tradicionales y pone en riesgo al pequeño productor tradicional y sus prácticas alrededor de las semillas, que involucran diversos significados de valor de uso, que fueron además simplificadas en el valor del cambio moviendo el objetivo, centrado en la cultura y la identidad a las actividades económicas. Esto redujo las áreas dedicadas a sistemas tradicionales, indujo la pérdida de agrodiversidad y provocó un rompimiento de la transmisión intergeneracional de conocimientos tradicionales, que deben ser restaurados.

La evaluación de las parcelas muestra que los custodios de semillas generan sistemas de gran diversidad biológica y congnitva y, por tanto, de alta complejidad, el mantener la producción tradicional, a través de arreglos de policultivos, cuya organización requiere una dinámica permanente de transformación, que permiten garantizar y mantener esta amplia diversidad en espacios pequeños. 
Este trabajo, a través del análisis del proceso de custodios de semillas, permite evidenciar el rompimiento en la transmisión de los conocimientos, que han sido inducida en el proceso de transformación productiva de la región (Martínez Alier, 1994), que no ha considerado los impactos negativos de la minería y la agricultura comercial en el impulso territorial.

El análisis mostró que la conservación de las semillas no puede estar distante de la conservación de la cultura, de la innovación con identidad y de la valoración de los conocimientos tradicionales; por lo tanto, el principal objetivo de generar una ruta de conservación, en este sentido, es posibilitar el vínculo entre conservación de las semillas y de los conocimientos tradicionales y generar estrategias de transmisión de conocimientos a las nuevas generaciones.

Es fundamental involucrar a los jóvenes en el proceso, con el fin de reducir los impactos generados por la pérdida de la diversidad genética en los sistemas productivos en el resguardo de Puracé.

Conflicto de intereses: El manuscrito fue preparado y revisado con la participación de todos los autores, quienes declaramos que no existe ningún conflicto de intereses que ponga en riesgo la validez de los resultados presentados.

\section{REFERENCIAS}

1. AGENCIA NACIONAL DE TIERRAS, ANT. 2018. Base de datos de Resguardos indígenas de Colombia (Colombia).

2. BADSTUE, L.; BELLON, M.; BERTHAUD, J.; RAMÍREZ, A.; FLORES, D.; JUÁREZ, X.; RAMÍREZ, F. 2006. Collective action for the conservation of on-farm genetic diversity in a center of crop diversity: An assessment of the role of traditional farmers' networks. Ed. IFPRI CAPRI (USA). 48p.

3. CABILDO INDÍGENA DE PURACÉ, CIP. 2020. Censo Indígena (Colombia).

4. CALDON, L.; BONILLA, C.; AGUILAR, G.; DIAZ, C.; QUILINDO; O.; LAME, M.; LAME, P.; NELLY, P.; AGUILAR, A. 2019. Relatoría conversatorio con los mayores sobre transición socieoecológica en el resguardo de Puracé. (Colombia). Cabildo indígena de Puracé.

5. CANO-CONTRERAS, E.J.C.; MEDINACELI, A.; DIAGO, O.L.S.; VILLAMAR, A.A. 2016. Código de Ética para la Investigación, la Investigación-Acción y la Colaboración Etnocientífica en América Latina. Versión Dos. Etnobiología. 13(4):5-6.

6. CASAS, A.; PARRA, F. 2007. Agrobiodiversidad, parientes silvestres y cultura. LEISA revista de agroecología. 23(2):5-8.

7. CASAS, A.; TORRES, J.; PARRA, F. 2016. Manejo de biodiversidad y evolución dirigida por las culturas del nuevo mundo. Ed. UNAM (México). 507p.
8. CHACÓN, X.; GARCÍA, M. 2016. Redes de custodios y guardianes de semillas y casas comunitarias de semillas nativas y criollas - Guía metodológica. Ed. SWISSAID y Corporación Biocomercio Sostenible (Colombia). 56p.

9. DZIB-AGUILAR, L.A.; ORTEGA-PACZKA, R.; SEGURACORREA, J.C. 2016. Conservación in situ y mejoramiento participativo de maíces criollos en la península de Yucatán. Tropical and Subtropical Agroecosystems (México). 19(1):51-59.

10. FORERO, J. 2010. El campesino colombiano: entre el protagonismo económico y el desconocimiento de la sociedad. Pontificia Universidad Javeriana (Colombia). 140p.

11. GUTIÉRREZ, L.; FITTING, E. 2016. The Red de Semillas Libres: Contesting Biohegemony in Colombia. Journal of Agrarian Change (Canadá). 16(4):711-719. https://doi.org/10.1111/joac.12161

12. HARLAN, J. 1971. Agricultural origins: centers and noncenters. Science. 174(4008):468-474.

13. HERMANN, M.; AMAYA, K.; LATOURNERIE, L.; CASTIÑEIRAS, L. 2009. ¿Cómo conservan los agricultores sus semillas en el trópico húmedo de Cuba, México y Perú? Bioversity International (Italia). 186p.

14. HOLT-GIMÉNEZ, E. 2006. Campesino a campesino: voices from Latin America's farmer to farmer movement for sustainable agriculture. food first books (Canadá). 225p.

15. INSTITUTO GEOGRÁFICO AGUSTÍN CODAZZI, IGAC. 2016. Base de datos catastral. Departamento del Cauca.

16. JIMENO, M.; TRIANA, A. 1985. Estado y minorías étnicas en Colombia. Ed. Cuadernos del Jaguar. (Colombia). 343p.

17. MALINOWSKI, B. 1986. Los argonautas del Pacífico occidental. Ed. Planeta de Agostini S.A. (España). 530p.

18. MARTÍNEZ ALIER, J. 1994. De la Ecologica al Ecologismo Popular. Ed. Icaria. Barcelona. 366p.

19. MAZABUEL, N. 1999. Plan de vida Resguardo de Puracé. Cabildo Indígena de Puracé. (Colombia). 125p.

20. PISO, A.; QUILINDO, O.; GÜAUÑA, J.; LAME, M.; CLOFE, M.; ESCOBAR, A.; CALDON, L.; ESCOBAR, S. 2019. Relatoría Taller perdida de semillas. (Colombia). Resguardo indígena de Puracé. S/E

21. PROCLAMA DEL CAUCA. 2016. Los custodios de semilla. Disponible desde Internet en:

https://www.proclamadelcauca.com/los-custodiossemillas-purace/ 
22. SANABRIA, O.L. 2001. Manejo vegetal en agroecosistemas tradicionales de Tierradentro, (Colombia). Editorial Universidad del Cauca. 144p.

23. SHIVA, V. 2020. ¿Quién alimenta realmente al mundo?: el fracaso de la agricultura industrial y la promesa de la agroecología. Ed. Capitán Swing Libros (España). 352p.

24. SLET'TO, B.I.; BRYAN, J.; TORRADO, M.; HALE, C.; BARRY, D. 2013. Territorialidad, mapeo participativo y política sobre los recursos naturales: la experiencia de América Latina. Cuadernos de Geografía: revista colombiana de geografía (Colombia). 22(2):193-209.

25. THIELE, G. 1999. Informal potato seed systems in the Andes: Why are they important and what should we do with them? World development (Bolivia). 27(1):83-99. https://doi.org/10.1016/S0305-750X(98)00128-4
26. TOLEDO, V.M. 2005. La memoria tradicional: la importancia agroecológica de los saberes locales. Leisa Revista de agroecología (México). 20(4):16-19.

27. VALENCIA, M.; MARTINEZ, J.P.; JAOQUÍ, S.; CASAS, A.F. 2017. Capítulo 8: historia de las transformaciones en ecosistemas paramunos: el caso del área de traslape del parque nacional natural Puracé. En: Perafán, A.; Elías, J. (Eds). Conflictos ambientales en ecosistemas estratégicos. América Latina y el Caribe. Siglos XIX y XX. Ed. UNAD (Colombia). p.113-133.

28. ZENT, S.; MAFFI, L. 2009. Vitality Index of Traditional Environmental Knowledge. World Wide Web electronic publication. Disponible desde Internet en: https://terralingua.org/ (con acceso el 01/02/2021). 